\title{
La France des Plantagenêts et l'emblème héraldique du lion dans les pièces historiques de Shakespeare
}

\section{Raymond Gardette}

\section{(2) OpenEdition \\ 1 Journals}

\section{Édition électronique}

URL : http://journals.openedition.org/shakespeare/566

DOI : 10.4000/shakespeare.566

ISSN : 2271-6424

Éditeur

Société Française Shakespeare

\section{Édition imprimée}

Date de publication : 1 novembre 2000

Pagination : 89-108

ISBN : 2-84269-407-4

\section{Référence électronique}

Raymond Gardette, «La France des Plantagenêts et l'emblème héraldique du lion dans les pièces historiques de Shakespeare ", Actes des congrès de la Société française Shakespeare [En ligne],

18 | 2000, mis en ligne le 01 novembre 2007, consulté le 22 avril 2019. URL : http://

journals.openedition.org/shakespeare/566 ; DOI : 10.4000/shakespeare.566 
La loi des armes contraint le roi de France, vaincu, à s'adresser à son vainqueur comme à son héritier légitime : "Notre très cher fils Henri, Roi d'Angleterre, Héritier de France» (Henry V, V.2.334-5). L'héritage de Richard Cœur de Lion retourne ainsi, à l'aube des temps modernes, à la Maison d'Angleterre. Néanmoins, les exploits du roi Henri V, dignes d'Édouard III et du Prince Noir (respectivement grand-père et père du roi Richard II, démis par le roi Henri IV, père d'Henri V) ne feront oublier que le temps d'un règne bref les conséquences néfastes de la rupture sacrilège de la chaîne de succession légitime chez les Plantagenêts.

$$
* * *
$$

$K$. Harry. No king of England, if not king of France.

(The Life of Henry the Fifth, II.2.190)

2nd Messenger. France is revolted from the English quite, Except some petty towns of no import.

The Dauphin Charles is crownèd king in Rheims ;

(The First Part of King Henry VI, I.1.90-2)

Les règnes entrecroisés d'Henri VI et d'Édouard IV signalent le déclin du royaume d'Angleterre et la perte des fiefs français, sur fond de guerres civiles (1422-1453). Henri $V$, roi exemplaire, n'est pas plus tôt mort qu'arrive de France la nouvelle de défaites successives :

Messenger. Guyenne, Compiègne, Rouen, Rheims, Orléans, Paris, Gisors, Poitiers are all quite lost.

( 1 Henry VI, I.1.60-1)

Les querelles entre factions vont mettre fin au rêve impérial du vainqueur d'Azincourt. L'Angleterre court le risque de ne plus être en mesure d'arborer la fleur de lys :

Messenger. Awake awake, English nobility!

[...]

Cropped are the flower-de-luces in your arms ;

Of England's coat, one half is cut away. 
Au rebours, l'épée providentielle de Jeanne la Pucelle «Decked with five flower-de-luces on each side» (1 Henry VI, I.3.78) - est le signe emblématique de la reconquête de son royaume par le dauphin Charles. Au cours de la bataille de Normandie (14371449), les enjeux dynastiques de la guerre qui fait rage sont, du côté anglais, subsumés sous des querelles d'ordre domestique. Parmi les héros d'Angleterre sacrifiés aux querelles dynastiques, Talbot (dont le fils s'avance au cœur de la mêlée, avant d'y trouver la mort, avec la vaillance «du lion affamé» - «like a hungry lion», 1 Henry VI, IV.7.7) - dénonce, avant sa propre fin héroïque, la trahison des vœux de chevalerie chez les lâches au combat:

Talbot. Hark, countrymen : either renew the fight

Or tear the lions out of England's coat.

Renounce your style ; give sheep in lions' stead.

Sheep run not half so treacherous from the wolf,

Or horse or oxen from the leopard,

As you fly from your oft-subduèd slaves.

(1 Henry VI, I.7.27-32)

En charge, jusqu'au sacrifice suprême, de la reconquête des territoires angevins, Talbot a juré à Salisbury mourant - à qui il accorde, légitimement, le patronyme «Plantagenêt» ${ }^{39}$ - de mettre le pays rebelle à feu et à sang, à la façon de Néron faisant un spectacle des villes incendiées (1 Henry VI, I.6.73-4). Dans un élan héroïque, aux tonalités impériales, le défenseur de l'héritage plantagenêt, fidèle à son serment, associe naturellement, le lion héraldique, qui orne ses oriflammes, aux fiefs à reconquérir. Perdre la France, ce serait accepter que la fleur de lys élimine le lion d'Angleterre, donc récuser le glorieux lignage, dont Richard Cœur de Lion est la figure emblématique. Le cœur du guerrier légendaire, conservé à Rouen, n'impose-t-il pas la reconquête de la ville, tombée aux mains de Jeanne la Pucelle?

Talbot. And I - as sure as English Henry lives, And as his father here was conqueror;

As sure as in this late betrayèd town Great Cœur-de-lion's heart was burièd So sure I swear to get the town or die. 
La guerre de France est dictée par la mémoire des ancêtres angevins. Aussi bien, les multiples victoires et conquêtes du grand capitaine (1 Henry VI, III.8.6-8) lui gagnent-elles la reconnaissance du roi Henri VI, lors de son couronnement à Paris ${ }^{40}$. Talbot, ayant déposé son épée aux pieds d'Henri, en signe d'humilité face à Dieu et à son roi, est immédiatement fait comte de Shrewsbury:

$$
K \text {. Henry. Therefore stand up, }
$$

\section{Talbot rises}

and for these good deserts We here create you Earl of Shrewsbury ;

L'adoubement de Talbot, en récompenses d'actes héroïques, est conforme au rite chevaleresque. Pour autant, le code de chevalerie est en danger, dans un contexte de guerre civile. La haine inexpiable entre les factions rivales, que le roi (soumis à de récurrentes crises de folie) laissera s'entredéchirer, met en danger le déroulement des guerres continentales. Richard d'York, dit Plantagenêt (fils de Richard, comte de Cambridge, traître à Henri V, pour des raisons dynastiques) s'oppose à Jean Beaufort, comte de Somerset, héritier de la maison de Lancastre. Shakespeare fait de leur rencontre (fictive), dans les jardins de l'École de Droit le Temple, le terminus a quo de la guerre des Deux Roses - où va s'accomplir la prophétie de l'évêque de Carlisle (Richard II, IV.12040), relancée par les prédictions d'Exeter, témoin des ravages causés par les dissensions dynastiques (1 Henry VI, III.1.186-97; IV.1.182-94). Le vieux prétendant Mortimer, avant de mourir, dans sa prison, a démontré à Richard d'York (qui le lui a demandé «In honour of a true Plantagenet», 1 Henry VI, II.5.52) que la couronne revient à l'héritier de la maison d'York. À sa suite, Henri VI va jusqu'à reconnaître la faiblesse de sa propre légitimité, lorsqu'il rétablit Richard dans ses privilèges dynastiques :

$K$. Henry. Stoop then, and set your knee against my foot.

\section{Richard kneels}

And in reguerdon of that duty done, I gird thee with the valiant sword of York. 
Rise, Richard, like a true Plantagenet, And rise created princely Duke of York.

( 1 Henry VI, III.1.173-7)

L'adoubement de Richard Plantagenêt relance la guerre dynastique - qui emportera et le roi et York, en même temps que leurs adversaires - au détriment des guerres en France. Talbot, que la lutte d'influence entre York et Somerset prive de renforts devant Bordeaux, en est la première victime :

Richard . Renownèd Talbot doth expect my aid, And I am louted by a traitor villain And cannot help the noble chevalier. God comfort him in this necessity ; If he miscarry, farewell wars in France !

(1 Henry VI, IV.3.12-6)

La mort de Talbot, entre autres incidents tragiques, cristallise la fin de la chevalerie, dont ne vont survivre que des rites artificiels; elle annonce des guerres inexpiables, au cœur d'un royaume menacé de mort, et la perte définitive des fiefs angevins. Le déclin de 1'Angleterre, dont l'enfant-roi devient un souverain faible et mystique (à l'opposé de son père, glorieux et mythique) est inexorable. Les querelles dynastiques vont entraîner la désintégration du corps politique. Le triptyque 1, 2, 3 Henry VI décrit les conséquences néfastes des injustices royales, de l'immaturité du peuple, et surtout, de la haine fratricide entre les nobles - les Lancastriens se réclamant d'une filiation directe, à partir du roi Henri $V$, les Yorkistes revendiquant le droit de promogéniture, qui les relie, grâce aux Mortimer, à Lionel, duc de Clarence. À l'inverse de son père, héroïque et glorieux, héritier du royaume de France, le jeune Henri VI ne réussira jamais à avoir raison des révoltes qui ensanglantent 1'Angletere, chez les grands du royaume comme dans le peuple. Son règne est l'interface, grotesque et tragique à la fois, de l'épopée henricienne. Le patronyme Plantagenêt, abondamment utilisé, ne renvoie pas, de façon paradoxale, à des conquêtes glorieuses, mais à des reniements, des complots, des assassinats, des révoltes populaires. Ces dernières sont, un moment, susceptibles de détruire la royauté même. Jack Cade, que soudoie le duc d'York, est une figure emblématique de l'anarchie, un Falstaff autorisé à mettre en ouvre ses instincts de destruction, dans un monde où les 
compagnons de taverne seraient investis de pouvoirs régaliens :

Cade. My father was a Mortimer -

Butcher (to his fellows). He was an honest man and a good bricklayer.

Cade. My mother was a Plantagenet -

Butcher (to his fellows). I knew her well, she was a midwife

$$
\text { ( } 2 \text { Henry VI, IV.2.40-4) }
$$

La faiblesse dynastique a pour conséquence une tragédie des vengeurs où chacun poursuit l'autre de sa vindicte, jusqu'à la mort. Les guerres fratricides, que ponctuent trois captures de rois (Henri VI, Édouard IV, à nouveau Henri VI - 3 Henry VI, III.1 ; IV.34 ; IV.7), trois renversements d'alliance (Warwick abandonne Édouard IV, de même que Clarence, avant de rallier à nouveau la maison d'York - 3 Henry VI, III.3 ; IV.1 ; V.1) conduisent aux défaites successives des Yorkistes et des Lancastriens, renouvelées dans le même ordre. Henri VI tente vainement de faire état de sa propre légitimité, face à York, au nom du lignage plantagenêt :

$K$. Henry. Ah, York, why seekest thou to depose me ?

Are we not both Plantagenets by birth,

And from two brothers lineally descent?

Suppose by right and equity thou be king -

Think'st thou that I will leave my kingly throne,

Wherein my grandsire and my father sat?

No - first shall war unpeople this my realm ;

Ay, and their colours, often borne in France,

And now in England to our heart's great sorrow, Shall be my winding-sheet.

(3 Henry VI, I.1.121-9)

L'allégorie in actis du corps du roi défunt recouvert des couleurs d'Angleterre, donc sacrifié à la cause dynastique, préfigure le régicide. La cause directe en sera le renoncement, par le roi, à la légitimité lancastrienne, imposée par la force des armes :

York. Confirm the crown to me and to mine heirs, And thou shalt reign in quiet while thou liv'st. 
K. Henry. I am content. Richard Plantagenet, Enjoy the kingdom after my decease.

La délégitimation du souverain par la violence, non plus de l'infidèle, comme dans la guerre sainte, mais contre sa propre lignée, va ouvrir la voie à une cruauté sans limite. Dans les multiples exactions et assassinats vont se mêler «les couleurs fatales» des deux «maisons en conflit». Henri VI en est le témoin impuissant. Incapable de résister aux forces déchaînées qui s'affrontent, il se réfugie dans une apologétique des victimes de la guerre dynastique. Dans sa complainte, face au spectacle d'un père qui assassine son fils, le lion ne symbolise plus, comme aux heures de gloire de la chevalerie, la force royale, mais uniquement la violence aveugle :

K. Henry. O piteous spectacle! O bloody times!

Whiles lions war and battle for their dens,

Poor harmless lambs abide their enmity.

Dans ce contexte, la mort d'York, après sa défaite par les forces lancastriennes, préfigure ce que l'on peut appeler le terminus ad quem du code chevaleresque. Improvisant un simulacre dérisoire des rites d'adoubement, la reine Marguerite pose sur la tête du duc une couronne en papier :

Q. Margaret. She puts a paper crown on York's head Ay, marry, sir, now looks he like a king, Ay, this is he that took King Henry's chair, And this is he was his adopted heir.

But how is it that great Plantagenet Is crowned so soon and broke his solemn oath ?

Off with the crown,

$$
\text { [...] }
$$

(She knocks it from his head)

and with the crown his head, And whilst we breathe, take time to do him dead. 
La parodie du couronnement, où le blasphème le dispute à la cruauté, signifie la fin des dynasties par lignage direct, dont sera victime, à son tour, la reine Marguerite. Le lien est disjoint entre les maisons rivales qui se réclament des Plantagenêts et l'emblématique héraldique royale : l'image du lion a perdu sa vertu sacramentelle ; elle ne traduit plus qu'un rapport de forces entre des armées engagées dans une lutte fratricide sans merci. C'est en vain qu'Henri VI refuse de s'incliner face à Édouard, au nom de ce qu'il croit être la «grâce» du lion royal :

$K$. Henry. No, Exeter, these graces challenge grace;

And when the lion fawns upon the lamb,

The lamb will never cease to follow him.

(3 Henry VI, IV.10.16-8)

L'heure n'est plus à la célébration de la divinité des rois, mais au châtiment qui rejaillit sur le petit-fils d'un roi usurpateur. Henri VI est une victime sacrificielle, au terme d'une guerre civile sans merci, qui entraîne aussi la perte des territoires français : sur lui, et sur le royaume d'Angleterre, rejaillit la faute de son grand-père régicide. Warwick, l'héroïque faiseur de rois, en mourant face à Édouard, rappelle les exigences de l'héritage légendaire, dont il fut l'un des derniers piliers:

Warwick. Thus yields the cedar to the axe's edge, Whose arms gave shelter to the princely eagle, Under whose shade the ramping lion slept, Whose top-branch over-peered Jove's spreading tree And kept low shrubs from winter's powerful wind.

$$
\text { (3 Henry VI, V.2.11-5) }{ }^{41}
$$

La mort de Warwick, comme celle de Talbot, est révélatrice de la lente désintégration de l'empire plantagenêt. Au moment où la maison d'York s'empare du trône d'Angleterre, elle ne met pas un terme aux guerres civiles. Chez Shakespeare, à l'instar des chroniqueurs du XVIe siècle, qui privilégient la vision providentielle de la guerre des Deux Roses, le règne de Richard III est l'ultime étape d'une descente aux enfers, où disparaissent, en même temps que le souvenir des heures de gloire des Plantagenêts, les codes de la Chevalerie. Édouard IV, qui reconquiert un trône grâce au sang versé par l'ennemi («Repurchased with the blood of enemies», 
3 Henry VI, V.7.2) - issu de la même souche angevine que sa propre lignée - se félicite de sa victoire sur ses vaillants adversaires («valiant foemen», 3 Henry VI, V.7.3), où l'on compte nombre de seigneurs de rang :

$K$. Edw. With them, the two brave bears, Warwick and Montague, That in their chains fettered the kingly lion And made the forest tremble when they roared. Thus have we swept suspicion from our seat And made our footstool of security.

\section{( 3 Henry VI, V.7.10-4)}

Il s'agit, par la force des armes, de désarmer et d'éliminer le roi en exercice. Le «lion royal», n'est plus évoqué en référence à un pouvoir quasi talismanique, inaliénable et sacré, mais seulement comme la marque d'un homme à abattre. L'emblème héraldique légendaire est devenu métaphore morte. L'exemple type de l'altération de la symbolique royale a d'ailleurs été donné par la sauvage et cruelle reine Marguerite soucieuse d'éliminer Humprey de Lancastre, duc de Gloucester (le plus jeune fils d'Henri IV) :

Q. Margaret. Small curs are not regarded when they grin, But great men tremble when the lion roars And Humphrey is not little man in England. First, note that he is near you in descent, And, should you fall, he is the next will mount.

(2 Henry VI, III.1.18-22)

C'en est fini, pour une maison comme pour l'autre, de la fidélité au lignage qui fit la grandeur de l'Angleterre. Lorsqu'il s'empare du trône, selon la méthode définie par l'épouse d'Henri VI, Édouard IV fait, en réalité, le lit du règne maléfique de son frère Richard, adoubé duc de Gloucester sur le champ de bataille, et qui ne fait nul mystère de ses intentions machiavéliques. L'imposture se traduira dans la supplantation du lignage, du sang, de la loi des armes par le meurtre. Au terme de la guerre des Deux Roses, la perte définitive des fiefs plantagenêts s'accompagne de la disparition des règles de la Chevalerie. 


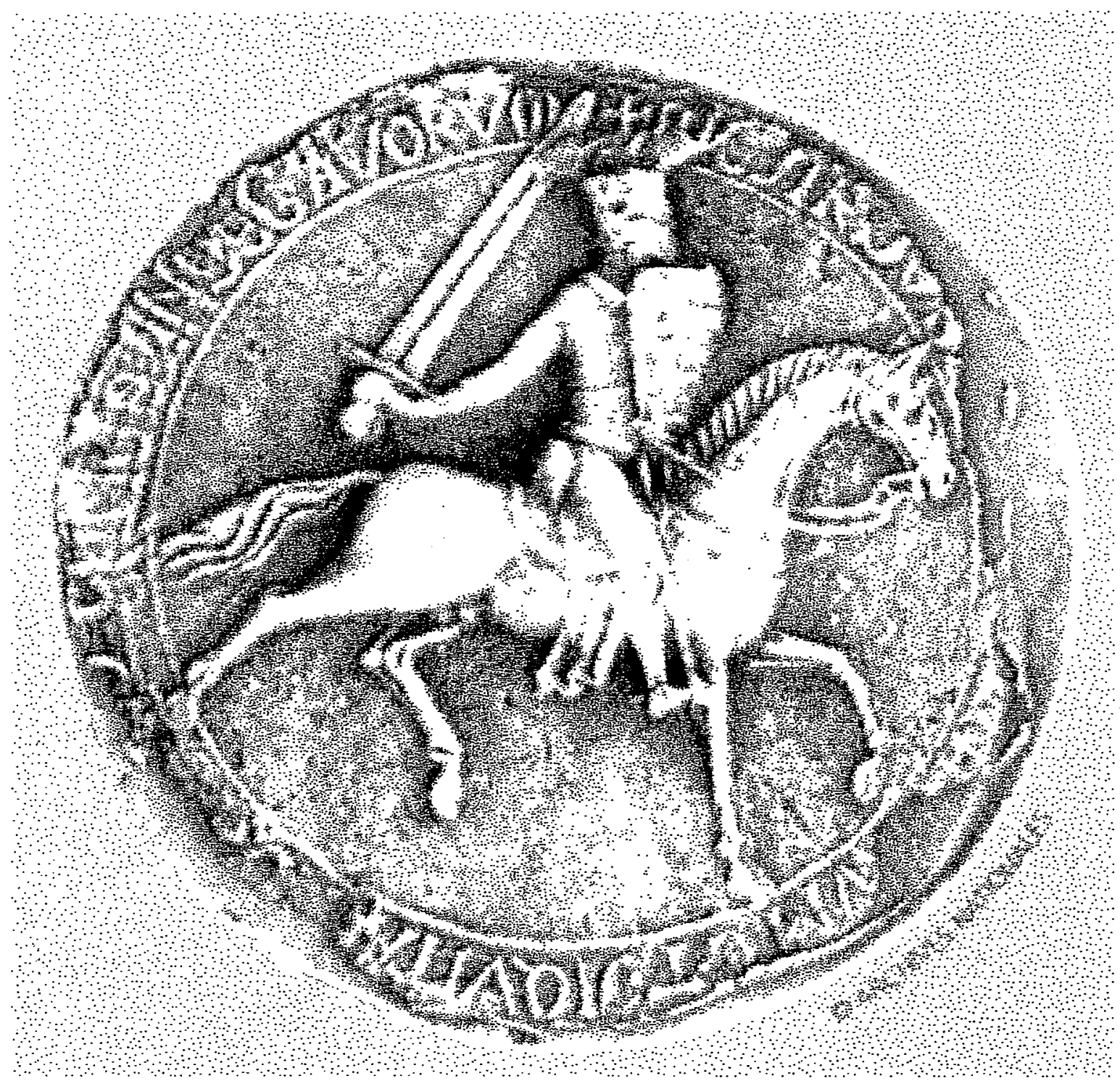

Sceau de Richard Coeur de Lion

deux lions rampants (verticaux) et affrontés

( (C) Archives Nationales, Paris) 



\section{$* * *$ \\ Cardinal Wolsey. \\ So looks the chafèd lion Upon the daring huntsman that has galled him, Then makes him nothing. \\ (Henry VIII, III.2.207-9) ${ }^{42}$}

L'ascendance angevine, leitmotiv des pièces historiques de Shakespeare, sous-tend les guerres dynastiques, cruelles et fratricides, qui ensanglantent l'Angleterre. Celles-ci ont pour cadre, sinon pour prétexte, l'empire légué par Richard Cœur de Lion à ses descendants - empire conquis, perdu, reconquis avant d'être abandonné, à l'image du trône royal. Le parallélisme qu'établit la saga shakespearienne entre les guerres en terre de France et sur le sol anglais est riche de perspectives ironiques. Du règne de Richard II à celui de Richard III, l'onction royale, que symbolise le lion héraldique, sans qu'il soit fait mention de l'héritage plantagenêt, est supplantée par l'opportunisme politique (policy), qui élimine toute référence à l'emblématique royale, pour, néanmoins, se recommander des Plantagenêts! Dans The Tragedy of King Richard the Second, l'épouse du roi lui rappelle, en vain, sa qualité royale, en utilisant le bestiaire emblématique :

Queen. The lion dying thrusteth forth his paw And wounds the earth, if nothing else, with rage To be o'erpowered ; and wilt thou, pupil-like, Take the correction, mildly kiss the rod, And fawn on rage with base humility, Which art a lion and the king of beasts ?

(Richard II, V.1.29-34)

Seule la force des armes contraint Richard II à se soumettre à une révolte usurpatrice, lui qui faisait du lion l'emblème d'un pouvoir royal, capable de contrer toute tentative de rebellion ${ }^{43}$. Dans The Tragedy of King Richard the Third, à l'inverse, il n'est nulle référence à l'emblématique héraldique du lion; par contre, jamais le patronyme Plantagenêt n'a eu plus d'usage qu'au moment où l'héritage d'Aliénor d'Aquitaine, depuis trois-cents ans aux mains de la dynastie (1154-1453), est perdu à jamais. Ainsi, Richard III, face à Anne, se décrit-il comme un Plantagenêt de meilleure extraction qu'Henri et Édouard qu'il a assassinés ; il met en scène le 
meurtre de Clarence sous le prétexte que celui-ci serait le seul meurtrier des héritiers légitimes du lignage ancestral ; il déguise son usurpation sous une légitimation par les Plantagenêts (Richard III, I.2.114-50; I.4.215-7; III.7.95-103). Le lignage est devenu synonyme de félonie, d'usurpation et d'assassinat (Richard III, IV.1.1-2 ; IV.4.17-30). La maison d'Anjou perd le royaume de France, en se livrant à l'auto-destruction, autre nom de la Tragédie.

Raymond GARDETTE Université Paris IV - Sorbonne 


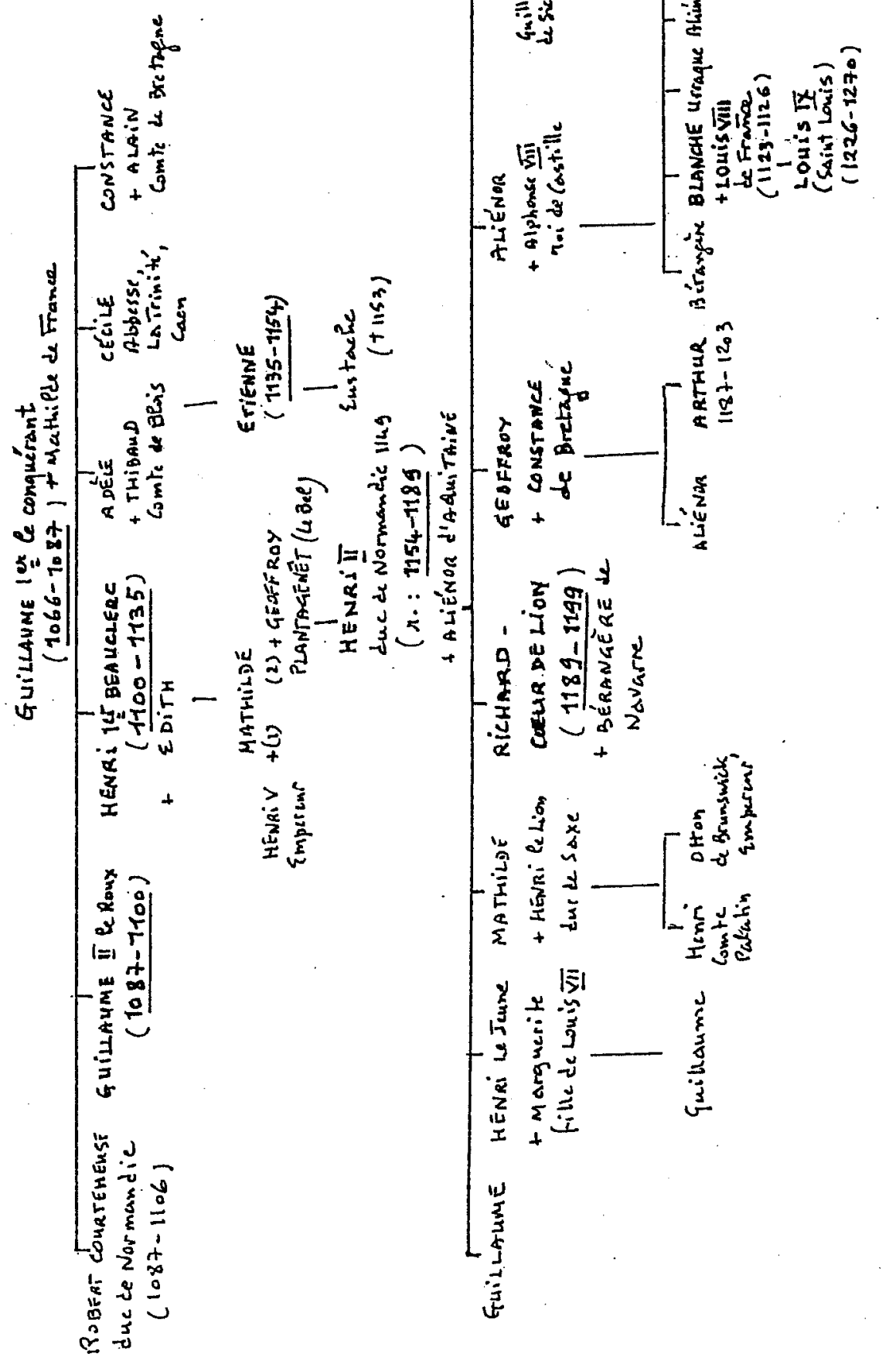

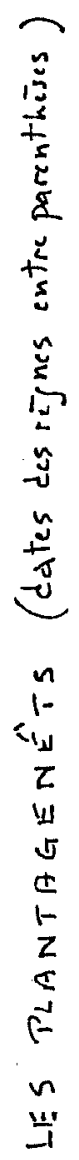




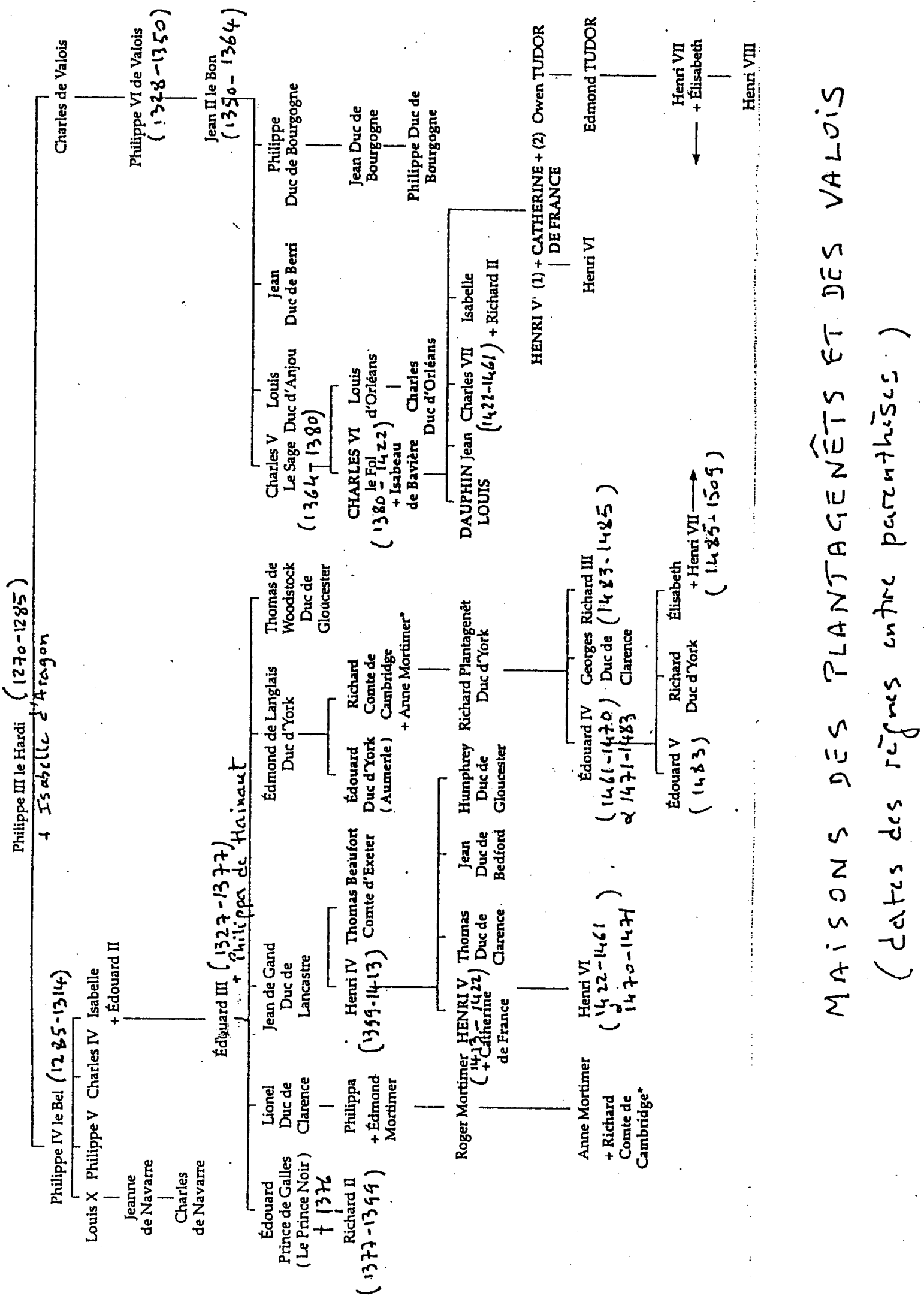




\section{NOTES}

${ }^{1}$ Édition de référence: William Shakespeare. The Complete Works, compact edition, eds. Stanley Wells \& Gary Taylor, Oxford, Clarendon Press, 1988.

${ }^{2}$ Les règnes des Plantagenêts sont regroupés en trois saisons in John Harvey, The Plantagenets, Londres, Fontana/Collins, 1967.

${ }^{3}$ Jean Flori, L'essor de la chevalerie. XIe-XIIe siècles, Genève, Droz, 1986, chap. III : «La remise des armes au XIe siècle: Adoubement ou investiture ?», p. 54-80 ; Marc Bloch, La Société féodale, Paris, Albin Michel, 1939, réimpr. Bibliothèque de l'évolution de l'humanité, 1994, chap. III : «La Chevalerie», I- «L'Adoubement», p. 435-41 ; Georges Duby, Les trois ordres ou l'imaginaire du féodalisme, Paris, Gallimard, 1978, réimpr. in Féodalité, Quarto Gallimard, Gallimard, 1996, II- «La Chevalerie», p. 758-74.

${ }^{4}$ Jean Flori, L'essor de la Chevalerie, p. 78.

${ }^{5}$ Traduction par Jean Flori, L'essor de la chevalerie, p. 60 \& note 22 (Orderic Vital, Historia ecclesiastica, VII, 16, ed. M. Chibnall, Oxford, 1973-78, vol. IV, p. 94-6).

${ }^{6}$ «[O]n amène des chevaux et des armes, que l'on distribue à chacun : cheval d'Espagne pour Geoffroy, rapide comme le vent. Armes incomparables, bouclier richement décoré de figures dorées, éperons d'or, heaume gemmé de pierres précieuses, lance de frêne, épée magnifique, provenant du trésor royal, forgée par l'artisan légendaire qui fit aussi Joyeuse, Durandal et Hauteclaire...». Commentaire de Jean Flori, L'essor de la chevalerie, p. 306 (à partir de Jean de Marmoutier, Chronique des contes d'Anjou et des seigneurs d'Amboise, éd. L. Halphen \& C. Poupardin, Paris, Auguste Picard, 1913, p. 178-80).

7 J. Flori, ibid., p. 265 (Jean de Marmoutier, p. 180); Michel Pastoureau, Traité d'héraldique, Picard éditeur, 1979, 3e éd. 1997, p. 143.

${ }^{8}$ Le Physiologus, compilation de l'apologétique alexandrienne (2e siècle - traduit, à partir d'un manuscrit grec perdu, en diverses langues orientales puis en latin, entre 1100 et 1400) introduisit le système typologique où des images zoologiques (animaux réels et imaginaires) étaient associées à des thèmes christologiques. Ce symbolisme animalier se retrouve dans les miséricordes des cathédrales, les encyclopédies et les bestiaires médiévaux. Parmi d'autres, le lion est un emblème du Christ : «The third attribute of the Lion is this: When the lioness brings forth her young, she brings it forth dead. But the Lioness watches over her cub until the third day, when the father comes and roars and breathes in its face and wakens it. So did the God and Father of the Universe waken the first born of 
all creation, our Lord Jesus Christ, his Son, from the dead. Well now spoke Jacob when he said of Judah the Lion's whelp: "Who shall rouse him up ?"»; Physiologus, Broadway Translations, Londres, George Routledge \& Sons, s. d., p. 188.

${ }^{9}$ Michel Pastoureau, Traité d'héraldique, p. 153.

${ }^{10}$ Le léopard est le fruit du croisement d'une lionne et d'un pard, mâle de la panthère ; Michel Pastoureau, Figures de l'héraldique, Découvertes Gallimard, Paris, Gallimard, 1996, p. 59.

11 «Richard, roi d'Angleterre, après avoir régné neuf ans, six mois et dix-neuf jours, fut frappé d'une flèche par Pierre Basile le 26 mars [1199], au château de Châlus, du territoire de Limoges, dans le duché d'Aquitaine»; Raoul de Diceto, Radulphi de Diceto de cani Lundonicusis opera historia, II.166. Citation et traduction de Jean Flori, Richard Cou r de Lion. Le Roi-chevalier, Biographie Payot, Paris, Éditions Payot \& Rivages, 1999 , p. 248.

12 À Foulques de Neuilly, prédicateur de la [troisième] croisade, lui reprochant «trois très mauvaises filles» [Superbe, Cupidité, Luxure], Richard aurait répondu : «Je donne donc ma fille Superbe aux orgueilleux Templiers, Cupidité aux moines de l'ordre de Citeaux et Luxure aux prélats des églises» ; citation et traduction de Jean Flori, Richard Couur de Lion, p. 468-9 (Roger de Hoveden [Howden], Chronica, ed. W. Stubbs, R.S., Londres, 1868-71, vol. IV, p. 76-7).

${ }^{13}$ J. Flori, L'essor de la chevalerie, p. 282-5, «l'archétype du roi chevalier».

14 Ibid., p. 126.

${ }^{15}$ Ibid., p. 479.

${ }^{16}$ L'emblème du lion est symbole de vaillance indomptable chez les chroniqueurs Richard de Devize, Raoul de Coggeshall, Giraud le Cambrien ; in Richard Cour de Lion, textes traduits et présentés par M. Brossard-Dandré \& G. Besson, 10/18, Paris, Christian Bourgois, p. 96, 99, 199, 201, 203, 289, 293. Le surnom «Cœur de Lion» (évoqué par Bernard Itier, in F. Arbellot (abbé), «Mort de Richard Cœur de Lion», Récits de l'histoire du Limousin, 1885) apparait dans Ambroise, L'Estoire de la guerre sainte (éd. G. Paris, 1897), récit des hauts faits d'armes du roi Richard, «le preuz reis, le quor de lion» (v. 2310); le lion, emblème des Plantagenêts, figure sur la bannière de Richard, au cours de la troisième croisade : «la veissiez tanz Turs acorre / Droit à la banière al lion» (v. 11526-7) ; citations d'Ambroise in J. Flori, L'essor de la chevalerie, p. 265.

${ }_{17}$ A. Crépin \& H.T. Dauby, Histoire de la littérature anglaise du Moyen Âge, Paris, Nathan, 1993, p. 117 ; J. Flori, Richard Cour de Lion, p. 266-7.

18 «The new seal, first used in May 1198, is important heraldically, for upon it the three lions, passant gardant of England, first appear», John Harvey, The Plantagenets, op. cit., p. 74. 
${ }^{19}$ Analyse de la succession par les femmes in Fanny Cosandey, La reine de France. Symbole et pouvoir, Paris, Gallimard, 2000, «Chapitre premier. La loi salique», p. 19-54.

${ }^{20}$ La filiation, en France, des Capétiens par les femmes est étudiée in Christiane Klapisch-Zuber, L'ombre des ancêtres. Essai sur l'imaginaire médiéval de la parenté, Fayard, «l'Esprit de la cité», 2000.

${ }^{21}$ «Quand le roi comprit qu'il était perdu, il légua à son frère Jean le royaume d'Angleterre et toutes ses autres terres...», Roger de Hoveden, Chronica, in Richard Cour de Lion, 10/18, p. 261 ; J. Flori, Richard Coeur de Lion, p. 234.

${ }_{22}$ J. Flori, Richard Cour de Lion, p. 463 (Roger de Hoveden, Chronica, IV, p. 97).

${ }^{23}$ «The same yere, Philip bastard sonne to king Richard, to whome his father had given the castell and honor of Coinacke, killed the vicount of Limoges, in revenge of his fathers death, who was slaine (as yee have heard) in besieging the castell of Chalus Cheverell», [Limoges est confondu avec le Duc d'Autriche dans The Troublesome Raigne of King John et dans le King John de Shakespeare]; Geoffrey Bullough (ed.), Narrative and Dramatic Sources of Shakespeare, Routledge \& Kegan Paul, 1962, vol. IV, p. 28.

${ }^{24}$ Par exemple, la lutte entre le roi Jean et le pape Innocent III dure 10 années (1205-16), alors qu'Arthur meurt en 1203; l'Église, qui appuie l'idée d'une invasion de l'Angleterre par Philippe Auguste (dont les navires sont détruits), finit par dénoncer l'expédition du dauphin, Jean ayant fait soumission à Rome ; Shakespeare, lui, implique Arthur dans les projets de Pandolphe pour faire plier le roi. La guerre civile dure deux ans après la réunion des nobles à Edmundsbury (1214) et c'est Jean qui utilise les mercenaires contre les barons, lesquels demandent à Louis de les aider en réclamant la couronne (1216); chez Shakespeare, ils prennent prétexte de l'invasion de l'Angleterre par le dauphin; King John, The New Cambridge Shakespeare, ed. L.A. Beaurline, Cambridge U.P., 1990, p. 199.

${ }^{25}$ John Gillingham, Richard Cour de Lion, Paris, Noésis, 1996, 4, «Crise familiale 1167-1174», p. 83-106.

${ }^{26}$ "Car il n'est qu'un bâtard de notre temps / Celui qui ne fleure pas la courtisanerie ; / Et c'est ce que je serai, que je la fleure ou non / Et pas seulement par l'habit et le blason...», Vie et mort du roi Jean, trad. Jean Michel Déprats, Paris, éd. Théâtre de Genneviliers, Théâtre/Public, 1999, p. 7.

${ }^{27}$ Shakespeare transfère à Angers la bataille de Mirebeau (1202), au cours de laquelle le roi Jean s'assura de la personne d'Arthur. Les détails de l'emprisonnement du jeune homme (qui disparut à Rouen, en avril 1203) sont empruntés à Holinshed (Bullough, p. 31-3). Le dramaturge s'inspire aussi du récit de la bataille d'Angers (1206), que Jean détruisit avant de la 
reconstruire : «But what will not an ordinarie man doo in the full tide of his furie; much more princes \& great men, whose anger is resembled to the roring of a lion, even upon light occasions oftentimes, to satisfie their unbrideled and brainsicke affections, which carrie them with a swift and full streame into such follies and dotages as are undecent for their degrees» (Bullough, p. 35).

28 Angers fut un des multiples enjeux de la lutte territoriale entre Philippe Auguste et Jean d'Angleterre, non le lieu d'exil d'Arthur, enjeu de la possession de la Bretagne. Le jeune prétendant, «soutenu par les chefs bretons [prit refuge] à la cour de Philippe Auguste», J. Flori, Richard Caur de Lion, p. 220 ; J. Gillingham, Richard Cour de Lion, p. 345-6.

${ }^{29}$ La jeune princesse semble évoquer l'héroïsme de Richard Cœur de Lion plutôt que le vol par Autriche (alias le vicomte Aimar de Limoges) du trophée (une peau de lion ?) du roi légendaire, après sa mort.

${ }^{30}$ Lors de son couronnement à Westminster, le dimanche 13 septembre 1189, Richard, après l'onction «prit (la couronne) lui-même et la tendit à l'archevêque (Baudoin)»; John Gillingham, Richard Cour de Lion, p. 181.

31 O. de Laborderie, «L'image de Richard Cour de Lion dans La Vie et la Mort du Roi Jean de William Shakespeare», in J.L. Nelson, Richard Cour de Lion in History and Myth, Londres, 1992, p. 141 sq.

32 Édition de référence : King Edward III, ed. Giorgio Melchiori, The New Cambridge Shakespeare, 1998.

33 Édouard III (roi : 1327-1377) revendique l'héritage dynastique de Guillaume de Normandie, couronné roi d'Angleterre en 1066, soit un peu plus de deux siècles et demi auparavant, et non cinq.

${ }^{34}$ Historiquement, l'adoubement du Prince Noir eut lieu à son arrivée en Normandie, en juillet 1346 et non avant la bataille de Crécy. Shakespeare utilise une indication d'Holinshed, selon laquelle le roi Édouard aurait refusé des renforts à son fils, le champ de bataille étant le seul lieu d'adoubement, par ordre du ciel, (Edward III, ed. G. Melchori, III.3.172-218) : «King Edward Tut, let him fight; we gave him arms today, I And he is labouring for a knighthood, man» (III.4.30-1).

${ }^{35}$ La capture du roi d'Écosse par Copland et son acte d'allégeance au roi d'Angleterre conduisent Édouard III à le faire chevalier : «King Edward Kneel therefore down. - Now rise King Edward's knight» (Edward III, V.1.94). Sur les libertés que Shakespeare prend avec l'Histoire, voir Giorgio Melchiori, ed. King Edward III, op. cit., «From sources to structure», p. 25 37.

36 La fleur de lys des Capétiens apparaît sur le sceau du fils aîné de Philippe Auguste, le futur Louis VIII en 1211. Peu à peu l'emblème, chargé de connotations chrétiennes, symbolise le rôle du roi de France, «médiateur entre le ciel et la terre, entre Dieu et les sujets de son royaume», Michel Pastoureau, Figures de l'héraldique, p. 101. 
37 «[A] lion roused»= un lion dressé, ou rampant évoque l'emblème gravé sur le bouclier de Richard Cœur de Lion en croisade.

38 L'image du lion souverain qui se dresse est celle du «lion rampant» sur le bouclier de Richard Cœur de Lion. La récurrence de l'appel au courage, sous cette forme métaphorique et héraldique, inscrit la question de légitimité au cour du discours dramatique.

39 Salisbury, dont le patronyme était Montacute, descendait de la maison d'Anjou, l'épouse du premier Lord Montacute étant Alice arrièrepetite-fille d'Édouard Ier.

${ }^{40}$ Henri VI est couronné roi de France à Paris, en 1431 ; il a alors neuf ans. (Le dauphin de France a été couronné, grâce à Jeanne d'Arc, sous le nom de Charles VII, à Reims, le 17 juillet 1429).

41 Warwick, comme Exeter devant Henri V (note 35), utilise l'image héraldique du lion rampant (dressé sur les pattes arrière), qui évoque le souvenir de Richard Cour de Lion en croisade.

${ }^{42}$ L'unique référence au symbole traditionnel dans Henry VIII, alors que Wolsey a trahi son souverain auprès du Pape, signale, ironiquement, la disparition de l'onction royale, issue d'un lignage mythique. Il reste que Wolsey se trompe quand il croit que le lion royal a perdu ses griffes.

${ }^{43}$ Face à Mowbray, Richard, utilisant le langage héraldique, oppose le lion au léopard (Richard II, I.1.174-5). Si l'écusson de Mowbray porte un lion rampant, alors il est fidèle à la tradition léguée par Richard Cœur de Lion, à son retour de croisade. Voir C.W. Scott-Giles, Shakespeare's Heraldry, Londres, 1950, 1971 ; cité in La tragédie du roi Richard II, trad. Jean-Michel Desprats, éd. Margaret Jones-Davies, Paris, Gallimard, Folio/Théâtre, 1968, p. 357. 